EVS27

Barcelona, Spain, November 17-20, 2013

\title{
How well can early adopters of electric vehicles be identified?
}

\author{
Patrick Plötz ${ }^{1}$, Till Gnann \\ ${ }^{1}$ Fraunhofer Institute for Systems and Innovation Research ISI, Karlsruhe, Germany, \\ patrick.ploetz@isi.fraunhofer.de
}

\begin{abstract}
Electric vehicles have recently been introduced to market in Europe. Policy makers as well as car manufacturers have great interest to understand the first group of electric vehicle users, the so-called 'early adopters'. Several studies have tried to determine the potential early adopters of electric vehicles from different angles. However, the number of available studies is limited and little is known about the actual statistical significance of characteristics for this important user group. Here we characterize the potential first users of electric vehicles from an economic perspective and ask which driving profiles make an electric vehicle cost-effective. To this end, we analyze a large database of German driving profiles and find the share of potential first users from different city sizes and statuses of employment. We first find the potential and in a second step study the statistical significance and robustness of the result by (1) performing Chi-square tests of the differences between potential early adopters and other vehicle owners and (2) varying important input parameters of our estimates. We find our characterization of the early adopters to be robust if battery prices and consumption costs are sufficiently favorable for a not too small group of users.
\end{abstract}

Keywords: Early adopter, user behaviour, driving profiles, market diffusion

\section{Introduction}

Electric vehicles (EVs) are an innovative propulsion technology that can help to reduce green house gas emissions from the transport sector as well as local emissions [1, 2]. In addition, electric propulsion is more efficient than propulsion via internal combustion engines and can support the shift from oil to other energy sources $[1,3]$. However, reliable estimates of the characteristics of future consumers of EVs are still limited [5, 6, 7] and the actual significance of these studies is disputable.
The goal of the present paper is to test the significance of different user groups' characterization as potential early adopters. For the identification of potential early adopters we follow the methodology of Biere et al. [9] and study the statistical significance of the approach in detail. The main point of our study is to determine whether a potential group of users in our sample shows higher likelihood of buying an EV than could be expected from their share of car ownership is more than a result of random fluctuations. 


\section{Data and Methods}

\subsection{Data and TCO calculation}

A large public data set of German driving behavior [4] is used for the identification of potential users of electric vehicles from an economical point of view. In the public survey, about 16,665 vehicles are included and their owners are included. For each vehicle the annual vehicle kilometers travelled and the share of city driving have been estimated by calculating the share of trips with average velocity below $18 \mathrm{~km} / \mathrm{h}$.

Based on technical parameters (e.g. fuel consumption or battery size) and economical parameters (for example fuel costs, battery price, and vehicle prize) the costs for vehicle purchase and operation can be estimated for each vehicle taking into account the user's specific driving profile. Both purchase and operation costs enter the total cost of ownership (TCO) which is used to find the cost optimal vehicle typ. The annual TCO for user $i$ are given by (cf. [8] for details):

$$
\mathrm{TCO}_{i}=I \cdot a_{n}(p)+365 L_{i}\left[s_{i} c^{\mathrm{ic}}+\left(1-s_{i}\right) c^{\mathrm{oc}}\right]
$$

Where $I$ denotes the investment for the given vehicle option, $a_{n}(p)$ is the annuity for an interest rate of $p$ over $n$ years (we choose $p=5 \%$ and $n=$ 8 years throughout), $L_{i}$ denotes the daily driving distance of user $i, s_{i}$ his or her share of inner city driving and $c^{\mathrm{ac}}\left(c^{\mathrm{oc}}\right)$ are the fuel consumption costs in inner (resp. outer city) driving. We assume all vehicles to be mid-size vehicles which is the largest group of cars (about $55 \%$ of stock) in Germany (see [8] and references therein). This is done for each vehicle in the data base and allows to state to which group users with high shares of cost-effective electric vehicles belong. In particular the data base contains information of the working status of the user (full time employee, par time employee, pensioner or not working) and the size of the municipality in which the user is living.

By assigning the TCO-optimal propulsion technology to each user profile we obtain the share of EV users from each of the 24 user groups and can compare them the share of this user group in all users. We use a chi square test to check the statistical significance of the deviation between the expected and observed share of EV users. For the former we assume the expected share of EV users to be simply similar to the overall share of users. The chi-square statistic is given by $\chi^{2}=\sum_{n}\left(o_{n}-e_{n}\right)^{2} / e_{n}$ where $e_{n}$ is the expected number of useres and $o_{n}$ the observed number of users in category $n$. For the present case of a two-by-two contingency table in the form $(a, b ; c, d)$ with a total sample size of $n$, this simplifies to [12]:

$$
\chi^{2}=\frac{n(a d-c b)^{2}}{(a+c)(b+d)(a+b)(c+d)} .
$$

The p-value is then given by (we use the fact that the cumulative distribution function of a $\chi^{2}$ distribution is a special case of the Gamma distribution and the latter is easily available for our numerical calculations):

$$
p=1-\Gamma\left(\chi^{2}, 1 / 2,2\right) .
$$

Where $\Gamma(x, a, b)$ denotes the cumulative distribution function (CDF) at $x$ of the Gamma distribution with parameters $a$ and $b$ (i.e. mean of the distribution is $a b$ and variance is $a b^{2}$ ). Different communities use different $p$-value thresholds for significance. Below we will use a value of $1 \%$ to indicate a threshold for statistical significance.

\subsection{Techno-economical Parameters}

The parameters are anticipated values for Germany and the year 2020. All vehicle specific parameters are given in Table 1.

Table 1: Techno-economical parameters

\begin{tabular}{|l|c|c|c|c|}
\hline Parameter & Gasoline & Diesel & BEV & PHEV \\
\hline $\begin{array}{l}\text { Investment } \\
\text { [Euro] }\end{array}$ & 19560 & 21560 & 18391 & 21529 \\
\hline $\begin{array}{l}\text { Battery size } \\
{[\mathrm{kWh}]}\end{array}$ & 0 & 0 & 24 & 10 \\
\hline $\begin{array}{l}\text { Fuel cons. } \\
\text { inner city } \\
{[\mathrm{l} / 100 \mathrm{~km}]}\end{array}$ & 8.5 & 6.3 & - & 7.0 \\
\hline $\begin{array}{l}\text { Fuel cons. } \\
\text { outer city } \\
{[\mathrm{l} / 100 \mathrm{~km}]}\end{array}$ & 5.7 & 4.5 & - & 6.2 \\
\hline $\begin{array}{l}\text { Elec. cons. } \\
\text { inner city } \\
{[\mathrm{kWh} / \mathrm{km}]}\end{array}$ & - & - & 0.182 & 0.182 \\
\hline $\begin{array}{l}\text { Elec. cons. } \\
\text { outer city } \\
{[\mathrm{kWh} / \mathrm{km}]}\end{array}$ & - & - & 0.207 & 0.207 \\
\hline $\begin{array}{l}\text { Electric } \\
\text { driving } \\
\text { share }\end{array}$ & $0 \%$ & $0 \%$ & $100 \%$ & $60 \%$ \\
\hline
\end{tabular}

Note the difference between inner city and outer city driving in fuel consumptions. This distinction acknowledges the fact that ICE vehicles are more 
efficient in constant mode of operation than in stop-and-go whereas electric vehicles are more efficient at low speeds and many stops. Furthermore, we assume an electric driving share of $60 \%$. We assume fuel prices of 1.6 Euro/l for gasoline, 1.5 Euro/l for diesel fuel and 0.2 Euro/kWh for electricity. VAT of $19 \%$ is added to all fuel prices. The battery price is rahter conservatively assumed as 400 Euros/kWh (including $19 \%$ VAT).

\section{Results}

\subsection{Identification of Early Adopters}

For given share of inner city driving and vehicle kilometers travelled, the cost-optimal propulsion technology can be estimated. Figure 1 shows these regions for Gasoline and Diesel vehicles as well BEVs and PHEVs taking into account the limited driving range of BEVs as well. Also shown are the VKT and inner city driving share of the users from the database under consideration (small crosses).

We observe from Figure 1 that users with high VKT are more likely to drive little within cities. Furthermore, EVs seem most cost-effective for a group of users with a minimal annual VKT but not too high VKT (where Diesel is cost-optimal). Overall, about $5.0 \%$ of all driving profiles are cost-effective for EVs in our analysis.

Finding the cost-optimal propulsion technology for each users driving profile we can analyze the status of employment and city size for all car users and potential EV users. This is shown in Figure 2. The determined potential EV users are not equally distributed among the 24 different user groups just as the car ownership is not equally distributed. Figure 2 shows the share of overall car users from the 24 different groups (dashed lines) together with share of EV users from each group among all EV users (solid lines). Our analysis indicates that most EV users in Germany can be expected to be full time or part time employees living in the small to medium sized $(0-50,000$ inhabitants $)$ municipalities. Contrary to common expectations, potential EV users are very unlikely (in terms of TCO) to live in large cities (with more than 100,000 inhabitants).

\subsection{Statistical Stability of Identification}

Observing the deviations between all users and EV users in Figure 2, we now turn to analyze their statistical significance. To this end, we construct two-by-two contingency tables for different sub samples

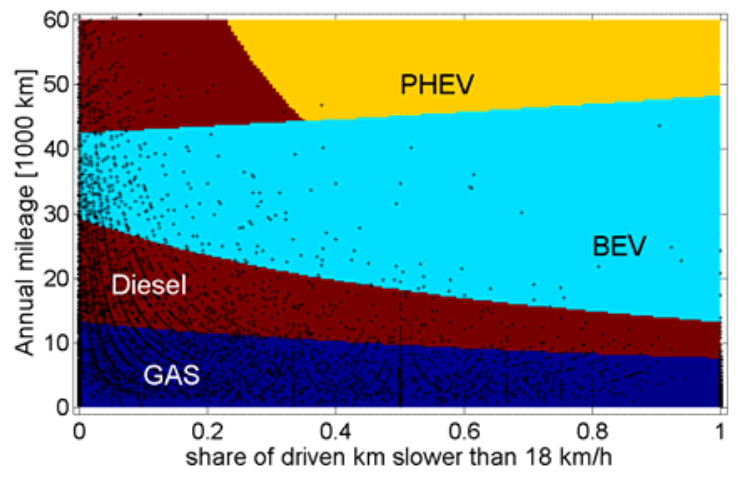

Figure1: Phase diagram of cost-optimal propulsion technology and position of sample users.

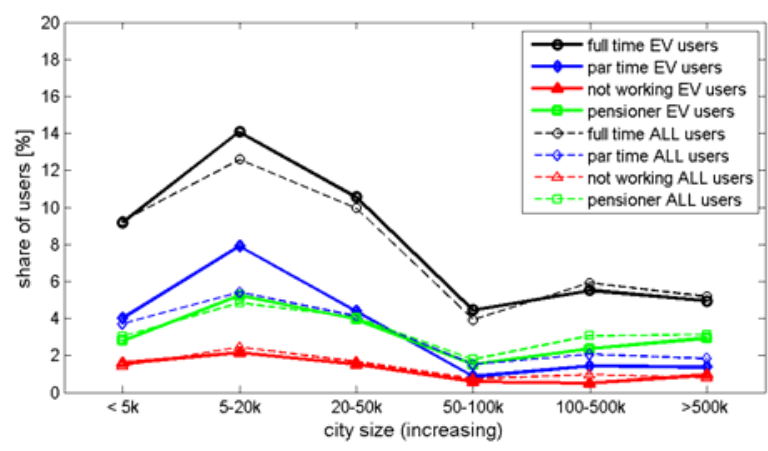

Figure 2: Shares of car users and potential EV users, i.e. early adopters, from the TCO estimate.

The latter holds since the inhabitants of larger cities in general show a low share in vehicle ownership (dashed line in Figure 2). Similarly, vehicles in general and (potential) EVs alike are mainly owned in small to medium sized cities (below 50,000 or below 100,000 inhabitants). It is important to note, that the distribution of vehicles is studied not the distribution of inhabitants in figure 2. About half of the German population lives in cities with more than 100,000 inhabitants but only about one quarter of the (privately owned) vehicles are registered there.

An important aspect for our further analysis are the differences between the dashed and solid lines in figure 2, i.e. the difference between expected and observed share of EV users from the 24 different user groups. For an analysis of different user groups, one can study all 24 user groups independently or aggregate them to larger groups. Here we will follow the first option (the latter has been taken up in [13]). 
with the absolute number of EV users from different employment statuses and different city sizes. That is we partially aggregate the groups such as "full time working EV user from city with more than 500,000 inhabitants". For all individual sub-groups we examined the share of users from different city sizes and employment status and compared the observed number of users with the expected number. We computed the chi-square statistics and the corresponding $p$-values to compute the probability that the observed deviations (i.e. the differences between solid and dashed lines in Figure 2) are only due to random effects. Figure 3 shows the calculated $p$-values as measure for statistical significance obtained from chi-square tests for varying battery prices. Shown are: full time working - top left, part time working - top right, not working - bottom left, pensioner - bottom right; all for varying battery prices in a range of $200-650$ Euro/kWh (we chose 400 Euro/kWh for figure 2).
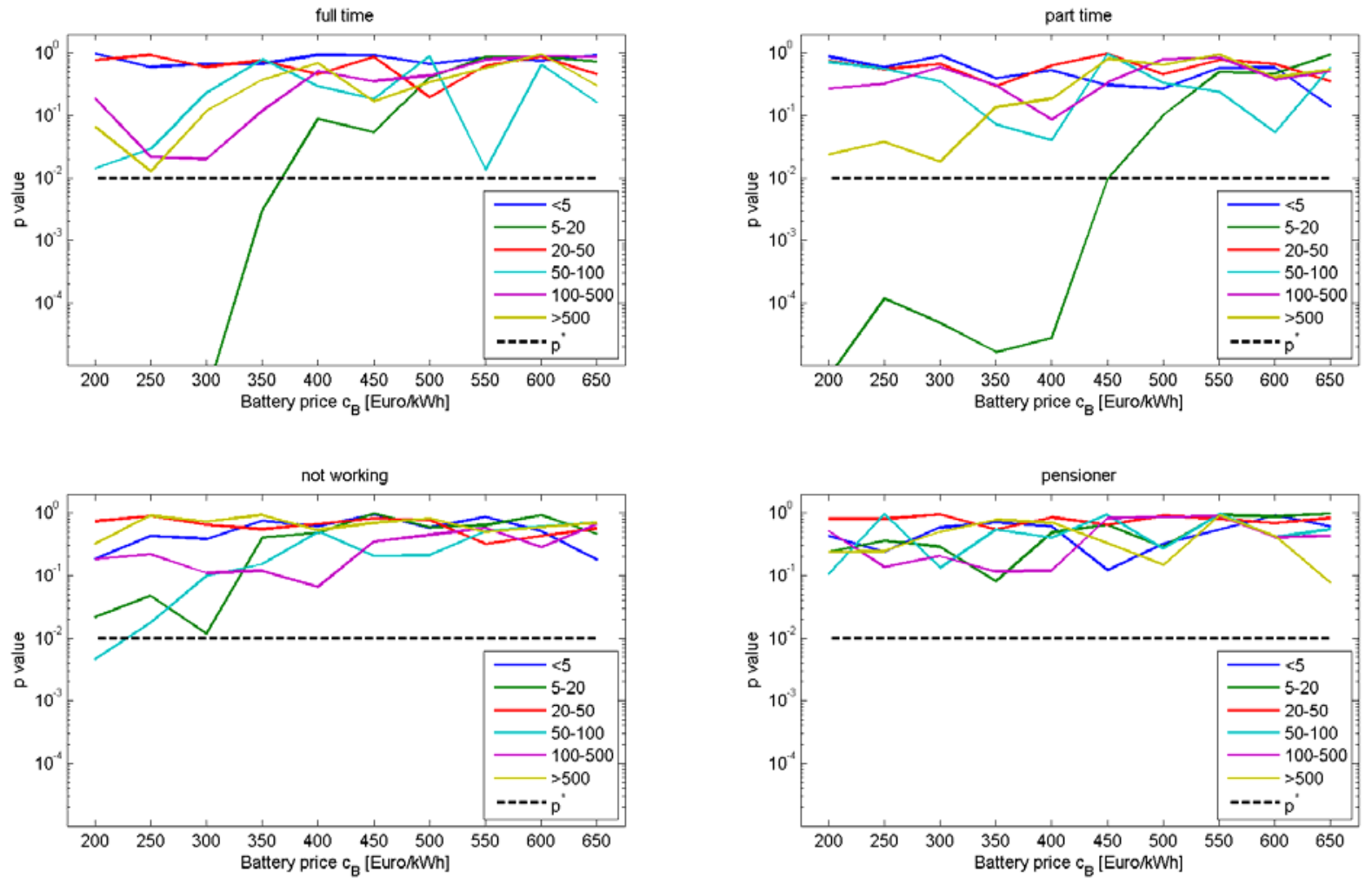

Figure 3: Statistical significance of differences between all users and potential EV users from different groups (full time working: top left, part time working: top right, not working: bottom left, pensioner: bottom right) as given by the $p$-value for differences due to pure random fluctuations. The critical value of $1 \%$ is marked as dashed line.

For the individual groups analyzed in Figure 3, we find only full time and part time employees from city sizes with 5,000 - 20,000 inhabitants to be more likely EV users than could be expected from the share of car ownership in general. For very cheap battery prices (below $300 \mathrm{Euro} / \mathrm{kWh}$ ) the number of potential early adopters from our TCO estimate becomes larger and more sub groups such as full and part time workers from major cities, come closer to deviate significantly from expectation by overall car ownership. However, the group of potential early adopters who are not working or on pension remains too small to draw reliable conclusions on their share of EV ownership.

\subsection{Sensitivity and Discussion}

\subsubsection{Inner city driving threshold}

Within the methodology presented above, the threshold for inner city driving is difficult to choose and to a certain degree arbitrary. To check the sensitivity of our results, we performed a similar analysis with different values for the threshold of inner city driving. Figure 4 shows the result of a calculation similar to the one presented above in Figure 3, except that the threshold for inner city driving has been set to $30 \mathrm{~km} / \mathrm{h}$. 

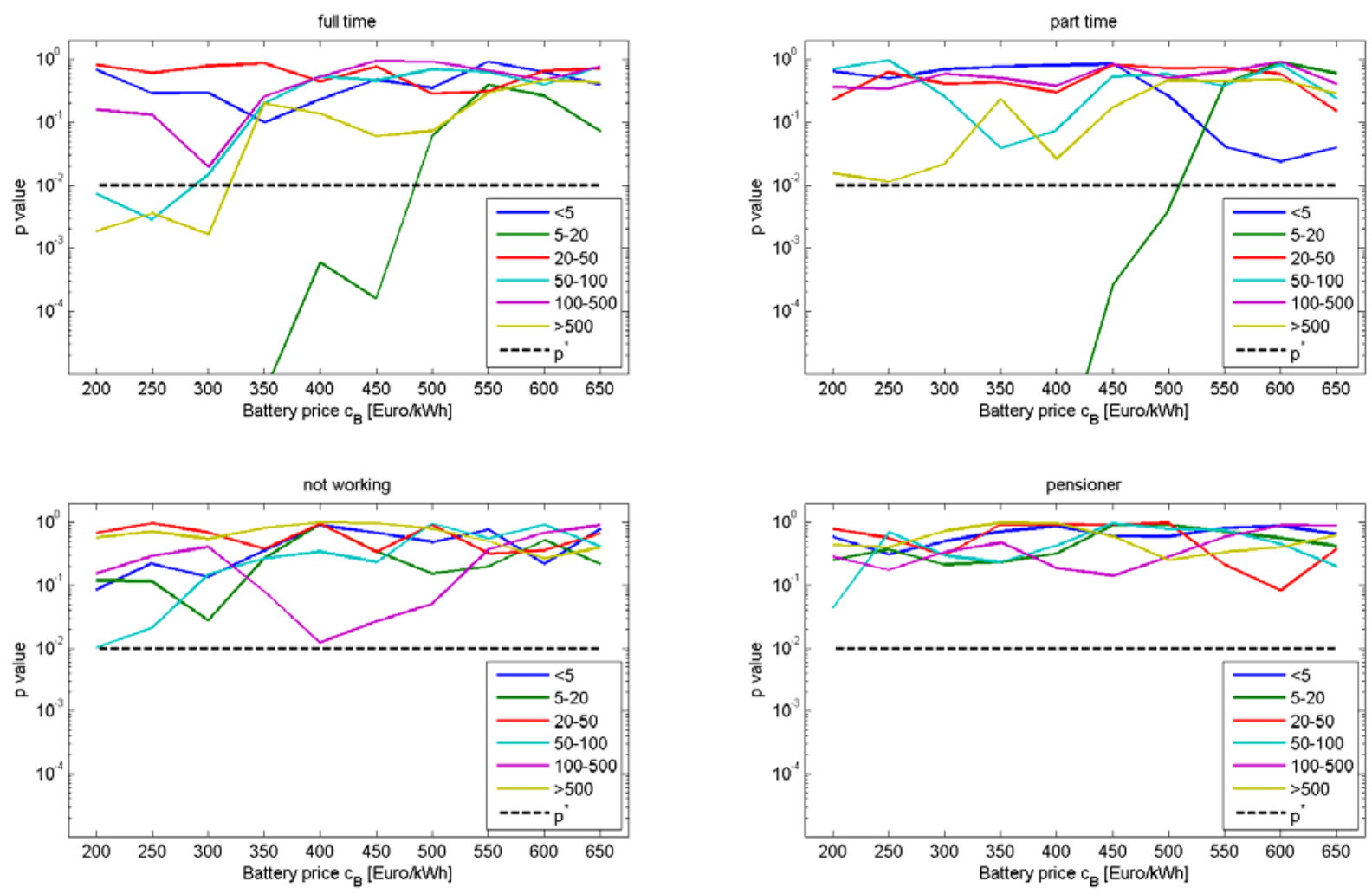

Figure 4: Statistical significance of differences between all users and potential EV users. All parameters as in figure 3, only the threshold for inner city driving has been set to $30 \mathrm{~km} / \mathrm{h}$. The critical value of $1 \%$ is marked as dashed line.
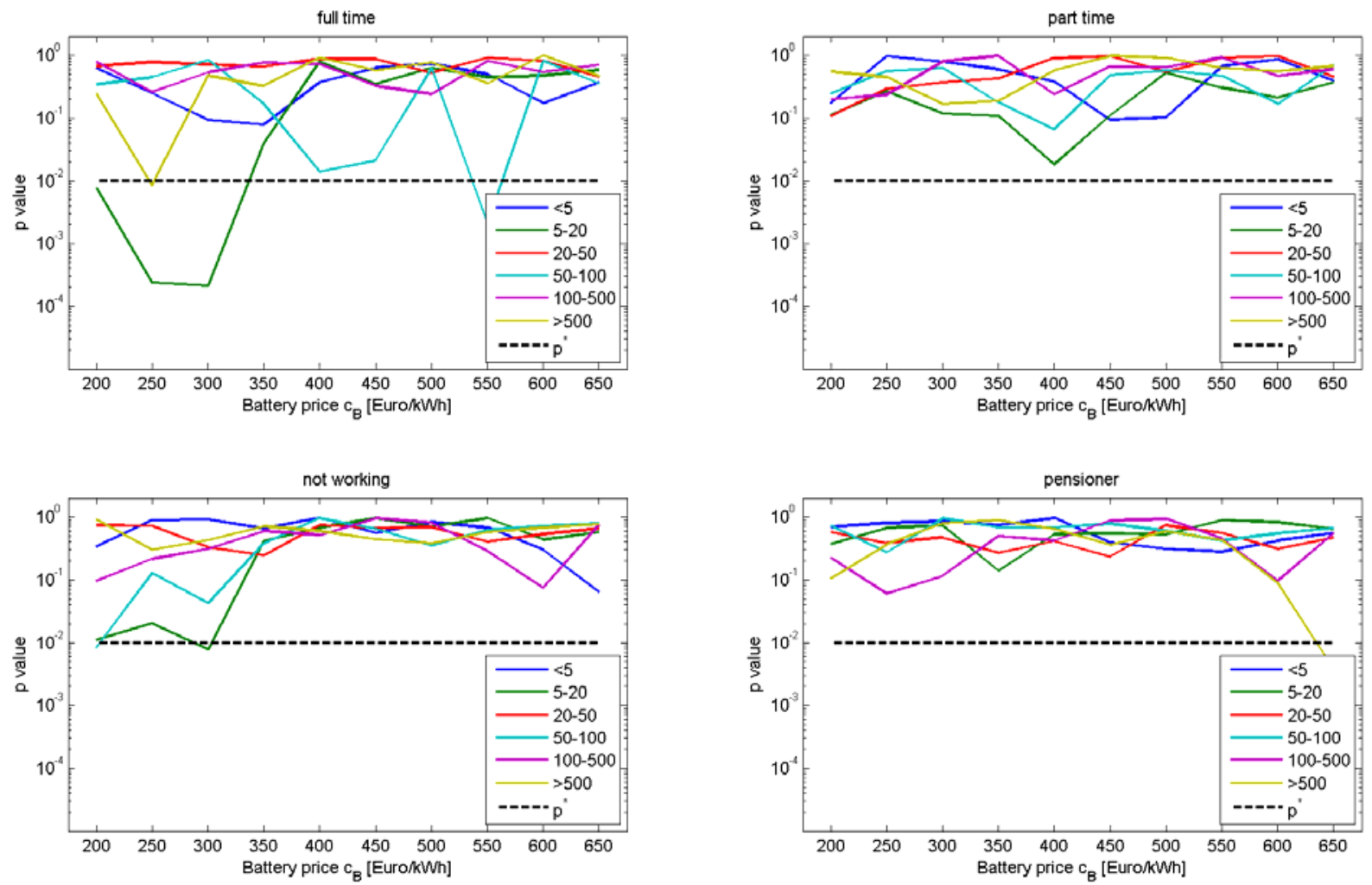

Figure 5: Statistical significance of differences between all users and potential EV users (see text). All parameters as in figure 3 , only the minimal number of trips per user is 3 . The critical value of $1 \%$ is marked as dashed line. 
Similar to the results in figure 3 , full time and part time employees from cities with 5,000 20,000 inhabitants show significantly higher shares in EV usage potential than could be expected from their share in car ownership in general (cf. top panels of Figure 4). Furthermore full and part time working employees from major German cities (with more than 500,000 inhabitants) are close the threshold of $1 \%$ for battery prices below 300 Euro/kWh.

\subsubsection{Minimum number of trips per user}

A further factor influencing the statistics of TCO-optimal vehicle choice is the limited amount of data for individual users. A close inspection of Figure 1 (or a density plot, cf. [13]) shows that a larger share of users seems to have a share of inner city driving of exactly one half. The reason is that many users perform a very limited number of trips on the day of observation. Only a single trip implies a share of inner city driving of either exactly one or zero, whereas two trips can lead to $0,1 / 2$ or 1 . To circumvent this statistical bias towards too simple fractions of inner city driving share, we excluded all driving profiles with less than three trips on the day of observation for a sensitivity analysis. The result is shown in Figure 5 with all parameters as in Figure 3, only users with less then three trips have been excluded (implying a smaller data set). Shown are: full time working top left, part time working - top right, not working - bottom left, pensioner - bottom right; all for varying battery prices in a range of 200 $650 \mathrm{Euro} / \mathrm{kWh}$.

The $p$-values in Figure 5 show again that very of the 24 users groups under consideration show significant deviations between the expected and observed share of EV users. Only full time working employees from cities with 5,000 20,000 inhabitants show significant (at the $1 \%$ level) deviations.

\subsubsection{Data sources}

We performed a similar analysis for a second set of private German driving profiles [10]. All passenger car driving profiles have been selected from this database and analyzed in terms of their total cost of ownership similar to the methodology outlined above (cf. [11] for details). The total set consists of 6339 individual driving profiles with one week of movements. A similar analysis [11] to the one presented here yields similar results: (1) full time employees living in small to medium sized municipalities are the largest group of users for which EVs are costeffective and (2) the EV shares of individual sub groups do not differ significantly from the shares of all car users but (3) the differences become significant when subgroups are merged. Overall, the analysis of a second data set shows slight differences in numbers but supports all three mentioned qualitative results.

A comparison with other studies of the potential early adopter of electric vehicles in Germany is consistent with our findings. Based on questionnaires and interviews with EV owners and people considering buying an EV in the near future, Dütschke and coworkers come to the conclusion that the early adopters in Germany can be expected to be full time working middle-aged men of higher education living in small to medium sized cities [14].

An important aspect in all tests for statistical significance is sample size. Here, we studied a large set of more than 15,000 driving profiles. However, constructing a subset of EV users (containing about $5 \%$ of all driving profiles) and dividing this into 24 user groups, we end up with rather small sub sample sizes ( $1 \%$ of $5 \%$ of $16,000$ is 8$)$. Thus, stronger results can be obtained from building larger, partially aggregated user groups (e.g. full or part time working). However, this in turn reduces the socio-demographic "resolution" of our analysis (cf. [13] for further discussion and results).

\section{Conclusions}

To summarize, the potential EV users are likely to be full or part time employees from small to medium sized cities. In detail more EV users are likely to come from these groups than expected from vehicle usage but it is not justified by our data and analysis to expect less (than based on their car ownership share) users from larger cities. The statistical significance of this estimate is strongly dependent on economical parameters since they influence the number of potential early adopters and thus the sub sample size.

\section{Acknowledgments}

PP wishes to express his gratitude to Elisabeth Dütschke, David Dallinger and David Biere for stimulating Discussions. 


\section{References}

[1] Bradley, T.H. and Frank, A.A. (2009): Design, demonstrations and sustainability impact assessments for plug-in hybrid electric vehicles. In: Renewable and Sustainable Energy Reviews 13, 115.

[2] Chan, C.C. (2007): The state of the art of electric, hybrid, and fuel cell vehicles. In: Proceedings of the IEEE, 95, 704.

[3] Thomas, C.E.S. (2012): How green are electric vehicles? International Journal of Hydrogen Energy, Volume 37, Issue 7, April 2012, Pages 6053-6062.

[4] infas and DLR. 2008. Mobilität in Deutschland (MiD) 2008. Bonn, Berlin: infas Institut für angewandte Sozialwissenschaft $\mathrm{GmbH}$, Deutsches Zentrum für Luft- und Raumfahrt e. V. (DLR).

[5] Anable, J.; Schuitema, G.; Skippon, S.; Kinnear, N. (2011): Who will adopt electric vehicles? A segmentation approach of UK consumers. In: ECEEE SUMMER STUDY ENERGY EFFICIENCY FIRST: THE FOUNDATION OF A LOW-CARBON SOCIETY, S. 1015-1026.

[6] Lieven, Theo; Mühlmeier, Silke; Henkel, Sven; Waller, Johann F. (2011): Who will buy electric cars? An empirical study in Germany. In: Transportation Research Part D: Transport and Environment 16 (3), S. 236-243.

[7] Santini, D.J.; Vyas, A.D. (2005): Suggestions for a New Vehicle Choice Model Simulating Advanced Vehicles Introduction Decisions (AVID): Structure and Coefficients. Report, Argonne National Laboratory. available online: http://www.transportation.anl.gov/pdfs/TA/350.pdf

[8] Plötz, P. Gnann T. and Wietschel, M. Total Ownership Cost Projection for the German Electric Vehicle Market with Implications for Future Market Shares and Electricity Demand. Enerday Dresden 2012.

[9] Biere, D.; Dallinger, D.; Wietschel, M. (2009): Ökonomische Analyse der Erstnutzer von Elektrofahrzeugen. Zeitschrift für Energiewirtschaft 33 (2009), Nr.2, pp.173-181.

[10] MOP (2010). Mobilitätspanel Deutschland 19942010. Institut für Verkehrswesen der Universität Karlsruhe (TH) and Clearingstelle Verkehr DLRInstituts für Verkehrsforschung: www.clearingstelleverkehr.de.
[11] Plötz, P., Gnann, T., Kühne, A. and Wietschel, M. (2013): Markthochlaufszenarien für Elektrofahrzeuge - Langfassung. Report, Fraunhofer ISI.

[12 Fahrmeir, L.; Künstler, R.; Pegeot, I.; Tutz, G. (2011). Statistik - Der Weg zur Daten-analyse. $7^{\text {th }}$ ed., Springer. 2011.

[13] Plötz, P., Gnann, T., (2013). Who should buy electric vehicles? - The potential early adopter from an economical perspective. In: Proceedings of the 2013 ECEEE summer study, Hyeres 2013.

[14] Plötz, P. Schneider, U. Globisch, J. and Dütschke., E. (2013): Who will buy electric vehicles? - Identifying the German early adopter. In preparation.

\section{Authors}

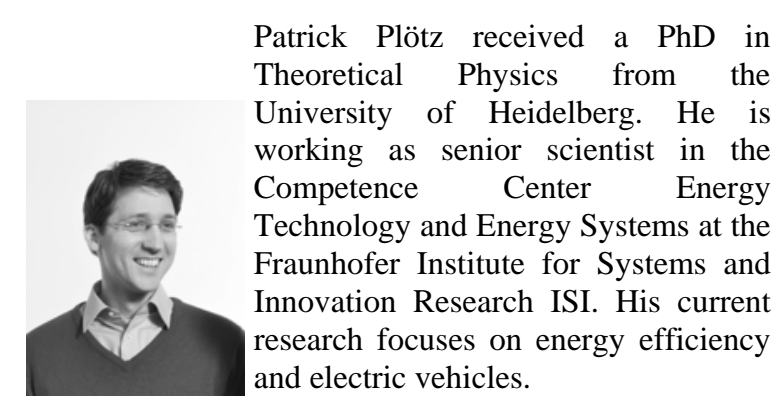

Till Gnann studied industrial engineering at the Karlsruhe Institute of Technology (KIT) and at the Politecnico di Milano. He is working

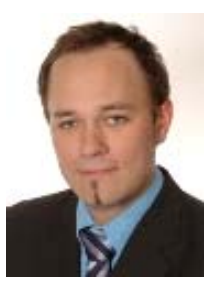
as scientist in the Competence Center Energy Technology and Energy Systems at the Fraunhofer Institute for Systems and Innovation Research ISI. His current research focuses on electric vehicles and their charging infrastructure. 